\title{
Impacting Underserved Communities as a GI Trainee
}

\author{
Alex Zhornitskiy ${ }^{1} \cdot$ Alexander Nguyen $^{2}$ · Jonathan Kaunitz ${ }^{3}$
}

Published online: 9 April 2020

(c) Springer Science+Business Media, LLC, part of Springer Nature 2020

\begin{abstract}
Gastroenterology fellowship programs commonly include VA and county hospitals whose patient populations consist of some of the most vulnerable and underserved populations in the country who have a multitude of socioeconomic hurdles that limit their ability to address ongoing medical issues, all while having a restricted political voice and receiving care in under-resourced clinical settings. Since trainees are integral to the care of these patients, they have available two approaches that can affect community and hospital-based change, namely quality improvement (QI) and healthcare advocacy. QI projects focused on optimizing colorectal cancer screening, and Helicobacter pylori testing/eradication can provide value at an institutional level. Healthcare advocacy can be approached through involvement in national gastroenterological associations or locally through means such as establishing a fellowship-based advocacy group similar to a journal club. Both routes enable trainees to positively impact underserved communities.
\end{abstract}

\section{Key Messages}

- GI fellows and medicine trainees can impact underserved communities through quality improvement and health advocacy.

- Initiating quality improvement projects involving colorectal cancer screening or Helicobacter pylori screening and eradication programs both have great significance in underserved populations.

- Healthcare advocacy can be accomplished through professional organizations or starting a fellowship-based group.

Alex Zhornitskiy

AZhornitskiy@mednet.ucla.edu

1 Department of Internal Medicine, Hospitalist Services, Ronald Reagan UCLA Medical Center, Los Angeles, CA, USA

2 Gastroenterology Fellowship Program, Vatche and Tamar Manoukian Division of Digestive Diseases, Ronald Reagan UCLA Medical Center, Los Angeles, CA, USA

3 Medical Service, West Los Angeles VAMC and Departments of Medicine and Surgery, David Geffen School of Medicine at UCLA, Los Angeles, CA, USA

\section{Introduction}

Medical students and trainees (residents or fellows) are often tasked with providing care for the most vulnerable and needy populations. When caring for underserved patients, one quickly realizes that the social burdens of poverty and lack of education substantially contribute to their underlying chronic, and occasionally acute, medical problems. Almost all gastroenterology fellowships incorporate either a county or VA hospital as part of their training, oftentimes both. The challenge that GI trainees often face is how to positively impact these populations amidst the finite resources available and their own limited time outside of their clinical responsibilities. Two possible routes that are accessible to any trainee are QI and health advocacy.

\section{Quality Improvement}

Trainees can begin by creating a QI project with the tangible goal of quantitating adherence to guideline-based preventative health measures. Next, the more challenging, and often most frustrating, part of any QI initiative can be approached: identifying the causes of sub-optimal adherence to medical care and developing a strategy to improve it. Ultimately, the goal is bridging the gap between medical knowledge and adherence [1]. There is not a universal solution, with most problems being primarily institution- and 
population-dependent. Given the added challenges to adherence experienced in underserved populations owing to lack of education, transportation, language barriers, or medical distrust, it is increasingly valuable for a trainee to establish such quality initiatives in an effort to improve health in marginalized communities. Establishing a partnership or mentor to help guide one's progress in understanding and approaching these problems is equally crucial to success.

\section{Colorectal Cancer Screening}

One project of remarkable importance is colorectal cancer (CRC) screening. In theory, it should be simple: perform screening tests on appropriate patients based on national guidelines and if positive, arrange for diagnostic evaluation. Nevertheless, like most preventative health initiatives, the challenge is in the implementation rather than in the knowledge. Unfortunately in underserved populations that are less likely to have access to regular medical care, CRC is initially manifest in its advanced stages [2]. As such, it is crucial to address the barriers to appropriate screening and intervention.

The most commonly used screening test for average risk individuals is annual fecal immunochemical testing (FIT) in patients 50-75 years old. A number of inefficiencies that arise when initiating screening via FIT and performing colonoscopy often occur; issues can include, but are not limited to, inappropriate use of FIT screening, institutional challenges of distributing and collecting FIT kits, patients' difficulty with returning FIT kits, institutional challenges with timely scheduling of colonoscopies for positive FIT tests, poor bowel prep, and patient misconceptions about colonoscopy [3]. Over the course of a 3-year fellowship, GI trainees can initiate a QI project to identify and address the specific inefficiencies of colorectal cancer screening within a community and hospital system that will identify opportunities to systematically improve care.

\section{Helicobacter Pylori Screening}

A similarly important investigation is the eradication of Helicobacter pylori (H. pylori). There is a well-documented association between $H$. pylori and both peptic ulcer disease (PUD), mucosa-associated lymphoid tissue (MALT) lymphoma, and gastric cancer. The link between $H$. pylori infection and gastric malignancy is particularly prevalent in US ethnic groups that are often underserved [4, 5]. Given significant antibiotic use leading to antibiotic resistance, new guidelines established in 2017 led to the transition from triple to quadruple therapy for most patients and confirmation of eradication as standard of care. Similar to colorectal cancer screening, the need for coordination between patients and providers significantly challenges adherence to the guidelines, particularly among underserved communities where time off of work and transportation are often significant hurdles, as are the cultural barriers that can exist with regard to taking four new medications when patients are otherwise asymptomatic. Initially, identifying $H$. pylori screening rates in patients with PUD, gastric cancer, and MALT lymphoma is crucial. Afterward, QI projects can be pursued to identify rates of quadruple therapy prescribed by providers (as opposed to triple therapy), rates of ordered confirmatory tests for eradication after treatment, or rates of confirmed eradication. Once deficiencies are identified, it will be crucial to investigate the barriers to adherence and establish interventions.

\section{Health Advocacy}

Health advocacy is another important component essential for improving healthcare delivery in underserved communities. Whereas trainees advocate on behalf of individual patients on a daily basis, few have any direct exposure to advocacy outside the confines of the hospital [6]. Advocacy for the underserved population can provide the greatest value as these patients, who, for a multitude of factors, have little to no political voice. Thus, trainee involvement in healthcare advocacy on a local, state, and national level is an opportunity to impact broad-scale positive change among those with the greatest needs.

As physicians, we are taught early on to place an emphasis on being a "patient advocate" and that our contributions should also extend outside the walls of the hospital or clinic. Issues pertaining to health insurance, government funding of medical care, physicians' abilities to prescribe certain medications, and affordability of medications are all matters that can directly influence our ability to provide high-quality medical care for a patient. Many of these issues are addressed in legislation considered by local, regional, state, or federal lawmakers who discuss them regularly. The consequences of passage or vetoing of this legislation is significant, potentially derailing even the most reasonable and medically supported plan of care such as using a biologic agent in a patient with severe ulcerative colitis. Furthermore, what is often overlooked but equally important in underserved populations are issues such as low-cost housing opportunities, food subsidies, mental health funding, and early childhood education, all of which significantly impact patient health in these communities.

There are multiple organizations that represent gastroenterological interests on a national stage including the American Gastroenterology Association (AGA), the American College of Gastroenterology (ACG), and the American Society for Gastrointestinal Endoscopy (ASGE). Within each organization is an advocacy component that can be 
joined, even as a trainee, with the goal of giving a voice to gastroenterologists on state and national policies that are affecting our patients. One good opportunity is through the Young Delegates, an advocacy branch of the AGA specific to current GI fellows and those who recently completed their fellowship training who are interested in GI healthcare advocacy.

On a local level, advocacy can be pursued through a variety of means, whether it be voicing concerns in person during City Council meetings or through submitting op-ed articles to local newspapers. While both are useful options, given the busy schedule of a GI trainee, the challenge can be with initiating efforts without the backing of an organization or support system for guidance. An approach that has worked at several institutions is to start a healthcare advocacy group within the residency or fellowship program. Meetings can be as infrequent as monthly with one or two individuals presenting and discussing a few relevant articles, or possibly pertinent election propositions, on topics affecting the underserved. The final step would be formulating a statement on behalf of the group and submitting it as an op-ed, a letter to an elected representative, or to the advocacy arm of one of the previously mentioned national organizations.

\section{Conclusion}

As a trainee, working in underserved communities is a privilege. One is endowed more responsibility given the increased patient load and limited resources as well as the opportunity to learn from pathologies that can be characteristic of these populations. Nonetheless, this is a vulnerable community with a limited social and economic safety net and a multitude of socioeconomic factors that impact a patient's health. Amidst their clinical responsibilities and time constraints, trainees have the capacity to influence large-scale change in these communities through QI and health advocacy.

\section{References}

1. Riley WJ, Moran JW, Corso LC, Beitsch LM, Bialek R, Cofsky A. Defining quality improvement in public health. J Public Health Manag Pract. 2010;16:5-7.

2. Ho C, Kornfield R, Vittinghoff E, Inadomi J, Yee H, Somsouk M. Late presentation of colorectal cancer in a vulnerable population. Am J Gastroenterol. 2013;108:466-470.

3. Ylitalo KR, Camp BG, Umstattd Meyer MR, et al. Barriers and facilitators of colorectal cancer screening in a federally qualified health center (FQHC). J Am Board Fam Med. 2019;32:180-190.

4. Kiely SC, Grzybicki DM, Sepulveda A, Capitano M, Toborowsky EJ. Chronic abdominal complaints and Helicobacter pylori in a Haitian population. J Health Care Poor Underserved. 2004;15:183-192.

5. Mohar A, Ley C, Guarner J, et al. Eradication rate of Helicobacter pylori in a Mexican population at high risk for gastric cancer and use of serology to assess cure. Am J Gastroenterol. 2002;97:2530-2535.

6. Luft LM. The essential role of physician as advocate: how and why we pass it on. Can Med Educ J. 2017;8:e109-e116. (eCollection 2017 June Review).

Publisher's Note Springer Nature remains neutral with regard to jurisdictional claims in published maps and institutional affiliations. 